\title{
A stress-based topology optimization method for heterogeneous structures
}

\author{
Conlan-Smith, Cian; James, Kai A.
}

Published in:

Structural and Multidisciplinary Optimization

Link to article, DOI:

10.1007/s00158-019-02207-9

Publication date:

2019

Document Version

Peer reviewed version

Link back to DTU Orbit

Citation (APA):

Conlan-Smith, C., \& James, K. A. (2019). A stress-based topology optimization method for heterogeneous structures. Structural and Multidisciplinary Optimization, 60(1), 167-183. https://doi.org/10.1007/s00158-01902207-9

\section{General rights}

Copyright and moral rights for the publications made accessible in the public portal are retained by the authors and/or other copyright owners and it is a condition of accessing publications that users recognise and abide by the legal requirements associated with these rights.

- Users may download and print one copy of any publication from the public portal for the purpose of private study or research.

- You may not further distribute the material or use it for any profit-making activity or commercial gain

- You may freely distribute the URL identifying the publication in the public portal

If you believe that this document breaches copyright please contact us providing details, and we will remove access to the work immediately and investigate your claim. 


\title{
A stress-based topology optimization method for heterogeneous structures
}

\author{
Cian Conlan-Smith ${ }^{1}$. Kai A. James ${ }^{2}$
}

\begin{abstract}
In this work we introduce a method to incorporate stress considerations in the topology optimization of heterogeneous structures. More specifically we focus on using functionally graded materials (FGMs) to produce compliant mechanism designs that are not susceptible to failure. Local material properties are achieved through interpolating between material properties of two or more base materials. Taking advantage of this method, we develop relationships between local Young's modulus and local yield stress, and apply stress criterion within the optimization problem. A solid isotropic material with penalization (SIMP) based method is applied where topology and local element material properties are optimized simultaneously. Sensitivities are calculated using an adjoint method, and derived in detail. Stress formulations implement the von Mises stress criterion, are relaxed in void regions, and are aggregated into a global form using a p-norm function to represent the maximum stress in the structure. For stress-constrained problems we maintain local stress control by imposing $m$ p-norm constraints on $m$ regions rather than a global constraint. Our method is first verified by solving the stress minimization of an L-bracket problem, and then multiple stress-constrained compliant mechanism problems are presented. Results suggest that good designs can be produced with the proposed method and that heterogeneous designs can outperform their homogeneous counterparts with respect to both mechanical advantage and reduced stress concentrations.
\end{abstract}

Keywords Topology Optimization · Functionally Graded Materials · Stress-Based design · Compliant Mechanism $\cdot$ Heterogeneous Structures

\section{Introduction}

Compliant mechanisms are commonly used as alternatives to traditional rigid link and pin joint mechanisms due to their various advantages such as reduced weight, reduced wear, and built-in restoring force. They also require no lubrication and no assembly. Compliant mechanisms achieve movement through bending of flexural members rather than rotation about pin joints, and as such they often comprise a single body. However to achieve this movement these mechanisms require the use of thin hinge-like compliant joints. These regions produce stress concentrations which limit the applicability of compliant mechanisms to low-load applications [32].

Topology optimization has been used extensively for compliant mechanism design since it was first proposed in [3]. This study used a Solid Isotropic Material with Penalization (SIMP) method but there has since been numerous compliant mechanism studies using different formulations such as the level set method [13,14]15], and sequential element rejection and admission (SERA) method [12]. There has also been a focus on heterogeneous material mechanisms where multiple materials are used to create a desired performance. Sigmund [17] used a SIMP based approach where each element in the design domain has two independent design variables which

\footnotetext{
${ }^{1}$ Technical University of Denmark, Department of Mechanical Engineering DK-2800 Lyngby, Denmark.

E-mail: cicosm@mek.dtu.dk

${ }^{2}$ University of Illinois Urbana-Champaign,

Department of Aerospace Engineering,

Urbana, Illinois, United States.

E-mail: kaijames@illinois.edu
} 
formulate a power-law with Hashin-Shtrikman interpolation scheme. Later Gaynor et al. [20] used a similar mutiphase SIMP method to produce two-material designs that were prototyped via 3D printing and experimentally verified. Yin et al. [18] used a density based peak function interpolation which requires only one design variable per element. Wang et al. and Alonso et al. implemented a level-set method [19] and a SERA method [16], respectively which were both applied to multi-material compliant mechanism designs.

By using multiple materials it is possible to reduce stress concentrations, however when discrete materials are used a stress concentration can be produced across the interface between materials [21]. Functionally graded materials (FGMs) are an alternative to using multiple discrete materials. FGMs are heterogeneous materials whose properties can vary based on spatial position, and can create a tailored gradation between two different materials [34]. This gradation is created by varying composition and microstructure with position, which can be represented as the mixing of two (or more) materials with different material properties [24]. By tailoring the gradation between materials in a structure, one can also tailor the material properties of the structure at a local level. This versatility of FGM designs makes them a perfect candidate for optimization problems. However, stress criteria are often not considered in the optimization of FGM design problems. As the material properties and micro-structure vary at a local level, yield stress in the structure will also vary at a local level.

In the literature there are some examples of topology optimization for FGM compliant mechanisms. Carbonari et al. [25,26] produced piezoelectric actuator designs using FGMs modelled as a mix of piezoelectric and nonpiezoelectric materials. This study used a density based material interpolation scheme for heterogeneous materials based on finite element shape functions, a method first introduced by Matsui and Terada [27]. Conlan-Smith et al. [1] developed a SIMP-based formulation for modelling FGMs in topology optimization problems. Here each element in the domain had two variables - one for topology optimization and another for material optimization. The local Young's modulus was derived from an equation relating the two independent variables and was similar to 3-phase topology optimization for discrete material distribution presented in [22,23].

There has been significant work in the past to develop methods of topology optimization that account for stress considerations. Duysinx and Bendsøe [4] implemented relaxed local stress constraints for porous composite materials based on the SIMP method. Implementing local stress constraints requires a large number of constraint functions which results in a high computational cost. Duysinx and Sigmund [5] extended this method into a single global constraint function using p-norm and p-mean functions. Parìs et al. [30] and Le et al. [6] presented methods that imposed regional stress constraints to maintain control over local stresses without the increased computational cost. Lee et al. [7] applied stress-based topology optimization to design dependant loading problems. Stump et al. [28] introduced a method of topology optimization for creating FGM distributions with a tailored von Mises stress field. Lipton has applied homogenization-based methods to the design of functionally graded and composite structures subject to stress constraints [46,47]. De Leon et al. [36] implemented a SIMP approach with a projection method and p-norm function to design compliant mechanisms which are not susceptible to failure. Chu et al. [35] developed a stress-based level set method for designing multi-material compliant mechanisms. These problems were solved using a multi-objective optimization method incorporating output displacement and compliance.

The authors previously found that FGM mechanisms can produce higher mechanical/geometric advantages compared to homogeneous and two material mechanisms as outlined in [1]. Additionally, this study compared the stress distributions in each design and found that increased mechanical performance also incurred higher stresses in the design. However, when formulating the optimization problem there were no considerations for stress. The current research will expand on previous work to develop a novel method for implementing stress-based design criteria in FGM structures. As material properties vary within the structure of FGMs, the yield stress will also vary at a local level. This paper outlines a novel framework to model the variation in local yield stress of heterogeneous structures where we interpolate between the properties of a number of base materials. Our method is employed in the topology optimization of FGM structures subject to stress considerations. The method is verified with the stress minimization of an L-Bracket problem which is compared to results present in the literature. We then extend this method to the design of compliant mechanisms subject to stress constraints. We hypothesise that by specifically selecting a range of materials with a favourable relationship between Young's modulus and yield stress we can produce compliant mechanism designs with the same or improved performance that are less susceptible to failure.

The subsequent sections are outlined as follows: topology optimization methods are covered in Section 2 . Stress-based formulations will be presented in Section 3, including details on sensitivity analysis. Results are presented in Section 4 which includes a model verification by studying a stress minimization L-bracket problem, 
and studies on two design domains for compliant mechanisms subject constraints on local stresses. Two yield stress interpolation functions are studied in each case with the two resource constraints introduced in [1]. For comparison purposes all design problems are also solved using a homogeneous material distribution. Section 5 provides additional examples which give more insight into the methods presented.

\section{Topology Optimization}

The purpose of topology optimization is to distribute material within a given design domain in order to produce an optimal structure. Optimality of the structure is defined by a given objective function which is subject to a number of constraints. The design domain, $\Omega$ is discretized into $N_{e}$ elements each with a set number of design variables $x_{e}$. All design variables can be compiled into a global vector represented as $\boldsymbol{x}$ throughout.

\subsection{SIMP formulation}

In this work, design problems will be solved using (a) homogeneous, and (b) functionally graded structures. The homogeneous method will follow a standard SIMP method where each element has one design variable representing a relative material density such that $0 \leq x_{e} \leq 1$. An element is part of the structure for $x_{e}=1$ and void for $x_{e}=0$. In order to achieve a clear 1-0 (solid-void) converged solution the local Young's modulus is penalized such that

$$
E_{e}=x_{e}^{p} E_{0}
$$

where $E_{0}$ is the Young's modulus of a solid element and $p$ is the penalization parameter. To converge to 1-0 designs, $p$ must be sufficiently large ( $p \geq 3$ ), however if $p$ is too large the optimizer has a tendency to converge to local minima [40,39]. Throughout this work, we take $p=3$. In actual practice a value of $x_{e}=0$ will result in 0 stiffness and cause singularities in the FEA solution. To overcome this problem we choose a minimum value of $x_{e}$ close to, but not equal to zero, such that $x_{\min } \leq x_{e} \leq 1$. Throughout this work $x_{\min }=10^{-3}$.

\subsection{FGM formulation}

For the FGM design problems we adopt a SIMP-based method introduced previously in [1]. Here, each element has two design variables $x_{0, e}$ and $x_{1, e}$, which independently control the material density (solid-void) and solid material stiffness respectively. Local Young's modulus in FGM designs is calculated using (2).

$$
E_{e}=x_{0, e^{p}}^{p}\left(E_{l}+x_{1, e}\left(E_{u}-E_{l}\right)\right)
$$

where $E_{l}$ and $E_{u}$ represent the lower and upper limits of the Young's modulus. The material density, $x_{0, e}$, is much like the design variables used in the homogeneous case (1) and as such is penalized (also with $p=3$ ) to promote a solid-void distribution. They also have the same bounds $x_{\min } \leq x_{0, e} \leq 1$, where $x_{\min }$ ensures there is some stiffness in void regions to avoid bad conditioning of the stiffness matrix. The material stiffness variable, $x_{1, e}$, is not penalized and is bounded by $0 \leq x_{1, e} \leq 1$, such that the optimizer is free to use any $x_{1, e}$ distribution without bias.

The most compliant material studied is lead and the stiffest is iron, whose Young's modulus values give the bounds $E_{l}$ and $E_{u}$ respectively. Lead and iron have yield stresses of $14 \mathrm{MPa}$ and $50 \mathrm{MPa}$ respectively [33]. In this work we will study two material relationships between Young's modulus and yield stress. Using these relationships, we can approximate the local yield stress as a function of the local Young's modulus, which is calculated in (2). We require sensitivities of these relationships in order to solve the optimization problem and as such it is necessary for $E_{e}-\sigma_{y}$ relationships to be smooth, continuous and differentiable for all $E_{e}$. Hence, a least squares method was used to interpolate between yield stress values of known materials. These relationships are shown in Figure 1. The relationships used in this study are taken as examples to show how the proposed methods can be applied. However, these methods can be applied to any relationship that is expressed as a continuous function. Note also that as a SIMP-based method is used it is necessary for the relationships to extend as low as the Young's modulus for void regions, $E_{\text {min }}=x_{\text {min }}^{p} E_{l}$.

Relationship A is an almost linear interpolation. Relationship B is based off a number of materials with a wide range of properties in order to show the versatility of the method. This gives rise to two distinctively different 


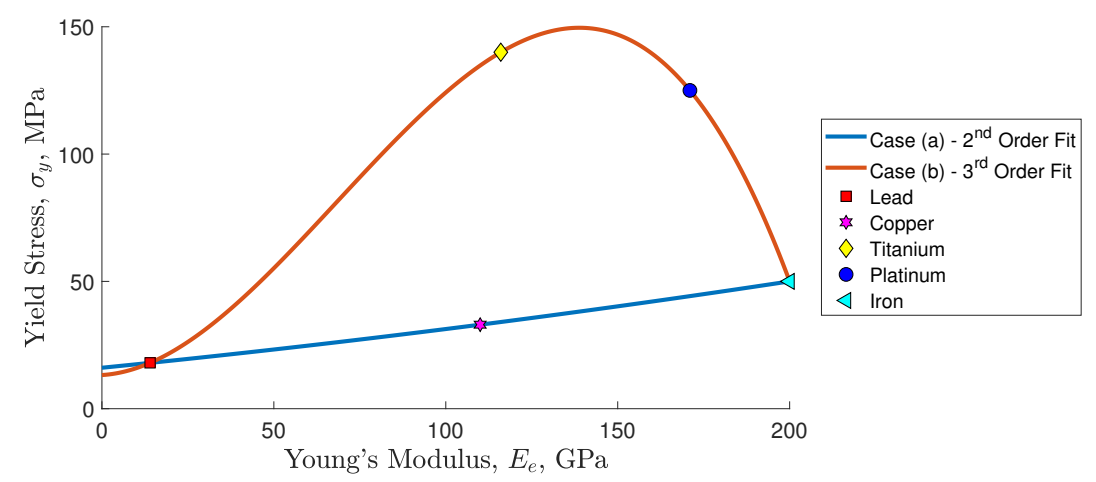

Fig. 1: Relationships between yield stress and Young's modulus for the FGM formulation.

relationships where both allow the optimizer to select material from the same range of Young's modulus values. The upper and lower bounds of the Young's modulus also have the same yield stress for relationships A and $\mathrm{B}$, however the yield stress of intermediate materials is drastically different between the two. Our hypothesis formulated in this study allows us to select material properties with a specific combination of Young's modulus and yield strength such that it can improve the stress distribution and performance of the structure.

\subsection{Density Filtering}

Filtering methods are used to achieve mesh independence, i.e. coarse and fine meshes should produce the same solution. We adopt a method of density filtering introduced by Bruns and Tortorelli [2]. Here, we define a filtering matrix, $\boldsymbol{W}$, such that

$$
\begin{aligned}
\tilde{\boldsymbol{x}} & =\boldsymbol{W} \boldsymbol{x} \\
\tilde{\boldsymbol{x}_{0}} & =\boldsymbol{W} \boldsymbol{x}_{0} \\
\tilde{\boldsymbol{x}_{1}} & =\boldsymbol{W} \boldsymbol{x}_{1}
\end{aligned}
$$

where $3 \mathrm{a}$ and $3 \mathrm{~b}$ correspond to homogeneous and FGM cases respectively, and $\tilde{\boldsymbol{x}}, \tilde{x_{0}}$, and $\tilde{\boldsymbol{x}_{1}}$ are the filtered design variables. The entries in $\boldsymbol{W}$ are calculated as

$$
W_{i j}=\frac{1}{\sum_{k=1}^{N_{r}} \bar{w}_{i k}} \bar{w}_{i j} \quad \text { where } \quad \bar{w}_{i j}=\max \left[0, r-d_{i j}\right]
$$

such that element $e_{j}$ contributes to the filtered value of element $e_{i}$ 's design variable at a weighting inversely proportional to the distance between centroids, $d_{i j}$, providing that the centroid of element $e_{j}$ is within a given filter radius, $r$, whose centre is coincident to $e_{i}$ 's centroid. For each centroid outside of $r$ the weighting is equal to zero. The rows of the matrix are then normalized such that the weights for each element within $r$ sum to 1 . Note that for the FGM formulation $\boldsymbol{x}_{0}$ and $\boldsymbol{x}_{1}$ are filtered independently and $\boldsymbol{W}$ is the same in each case.

\subsection{Finite Element Analysis}

Linear finite element analysis is the standard for solving problems with metallic materials. These materials experience a linear elastic force-displacement relationship provided the material does not yield. This condition can be enforced with the use of a stress constraint such that the maximum stress in the structure does not exceed a prescribed value. The stress formulation adopted in this work is discussed in detail in the next section. Given an external force distribution in the form a vector, $\mathbf{f}$, a nodal displacement vector, $\mathbf{u}$, can be determined via the following governing equation

$$
\mathbf{R}=\mathbf{K}(\boldsymbol{x}) \mathbf{u}-\mathbf{f}=0
$$


where $\mathbf{K}$ is the global stiffness matrix, which is a function of the design variables, $\boldsymbol{x}$, and is a global assembly of element stiffness matrices $\mathbf{k}_{e}$. Element stiffness matrices are calculated as follows

$$
\mathbf{k}_{e}=\int_{\Omega_{e}} \mathbf{B}^{T} \mathbf{D B} d \Omega_{e}
$$

where $\mathbf{B}$ is a strain-displacement matrix containing spatial derivatives of the shape functions, $\mathbf{N}$, with respect to the deformed nodal coordinates, $\mathbf{x}$, and expressed in Voigt form. The constitutive matrix assumes plane stress and is represented by $\mathbf{D}$. The expression $\int_{\Omega_{e}} d \Omega_{e}$ represents an integral over the element volume. Matrices $\mathbf{B}$ and $\mathbf{D}$ are calculated in as

$$
\begin{gathered}
\mathbf{B}=\frac{\partial \mathbf{N}}{\partial \mathbf{x}} \\
\mathbf{D}=\frac{E_{e}}{1-v^{2}}\left[\begin{array}{ccc}
1 & v & 0 \\
v & 1 & 0 \\
0 & 0 & \frac{1}{2}(1-v)
\end{array}\right]
\end{gathered}
$$

where $E_{e}$ is the Young's modulus of the element and $v$ is the Poisson's ratio. Poisson's ratio was taken to be 0.35 throughout. Element stresses can then be determined using the strains, $\boldsymbol{\varepsilon}$.

$$
\boldsymbol{\sigma}=\mathbf{D} \boldsymbol{\varepsilon}=\mathbf{D B} \mathbf{u}_{e}
$$

\section{Stress Based Design}

Compliant mechanisms incorporate thin compliant joints to achieve movement, however, these thin members also create stress concentrations in the design. Stress concentrations are areas of increased stress caused by irregular geometry such as sudden changes in thickness, holes or sharp corners. For this work we will implement stress formulations based on the von Mises yield criterion.

\subsection{The von Mises Stress Formulation}

The von Mises yield criterion is an empirical method of determining whether a material will yield when subjected to complex loading. Von Mises proposed the method in $1913[8]$ and in 1931 Taylor and Quinney [9] found that the method is a more accurate predictor for yielding in metals than any previous suggested methods, such as Tresca's maximum yield stress criterion [10]. The von Mises stress criterion states that a material will yield if the von Mises stress attains the material's yield stress. The von Mises stress for plane stress conditions is defined as

$$
\sigma_{V M}=\sqrt{\sigma_{x x}^{2}-\sigma_{x x} \sigma_{y y}+\sigma_{y y}^{2}+3 \sigma_{x y}^{2}}
$$

where $\sigma_{x x}, \sigma_{y y}, \sigma_{x y}$ are calculated as the entries in (9). The von Mises stress is calculated at the four Gauss points of an element and averaged to obtain the average von Mises stress within the element, $\bar{\sigma}_{V M}$.

\subsection{The Consistent Stress Formulation}

The von Mises stress criterion states that a material will yield if the von Mises stress attains the yield stress. Hence, to avoid yielding we must ensure the von Mises stress for each element in the design domain is less than the local yield stress.

$$
\begin{gathered}
\bar{\sigma}_{V M} \leq \sigma_{y} \\
\bar{\sigma}_{V M} \leq \sigma_{y, E_{e}}
\end{gathered}
$$

where (11a) represents the homogeneous case with a constant yield stress, $\sigma_{y}$, and (11b) represents the FGM case where $\sigma_{y, E_{e}}$ is the local yield stress evaluated at the Young's modulus, $E_{e}$, as defined in Figure 1 In this way we 
account for the fact that the different base materials have different yield limits.

Constraint relaxation techniques are employed to circumvent the singularity problem associated with stress constraints. This problem has been studied extensively for truss structures [41, 42, 43, 44] but has equal importance for the continuum case $4,5,29$. The problem arises if the design space contains a degenerate region within which the global optimum is located. By relaxing the stress constraint we generate a smooth, continuous design space. This is similar to the approach adopted in the SIMP-method to create a smooth design space by allowing intermediate element densities in place of a discrete 1-0 distribution. Stresses are relaxed as follows

$$
\begin{gathered}
\bar{\sigma}=x_{e} \eta^{\eta} \frac{\bar{\sigma}_{V M}}{\sigma_{y}} \\
\bar{\sigma}=x_{0, e}{ }^{\eta} \frac{\bar{\sigma}_{V M}}{\sigma_{y, E_{e}}}
\end{gathered}
$$

where $\bar{\sigma} \leq 1$ to satisfy the von Mises yield criterion. In using this formulation, stresses are relaxed in the element by multiplying by the material density raised to the exponential $\eta$, which is chosen to be 0.5 . By relaxing the von Mises stress in this manner, high stresses in void regions do not lead to high values of $\bar{\sigma}$.

The function in (12) is calculated for each element in the design mesh. However, we are only interested in the element where stress is highest and to enforce 12 as a constraint on each element will be computationally expensive. Hence we use an aggregation technique based on the p-norm function to find the element with maximum stress which gives the final expression for homogeneous (13a) and FGM (13b) designs as

$$
\begin{gathered}
\sigma_{\max }=\max (\overline{\boldsymbol{\sigma}}) \simeq\left[\sum_{e=1}^{N_{e}}\left(x_{e} \eta^{\eta} \frac{\bar{\sigma}_{V M}}{\sigma_{y}}\right)^{\zeta}\right]^{\frac{1}{\zeta}} \\
\sigma_{\max }=\max (\bar{\sigma}) \simeq\left[\sum_{e=1}^{N_{e}}\left(x_{0, e} \eta \frac{\bar{\sigma}_{V M}}{\sigma_{y, E_{e}}}\right)^{\zeta}\right]^{\frac{1}{\zeta}} .
\end{gathered}
$$

In this work, we formulate stress-based design in two ways - to minimize the maximum stress, and to impose stress constraints such that $\sigma_{\max }$ does not exceed the yield stress. For minimizing the stress the objective function is to minimize the function given in (13). When a stress constraint is applied, one wants to ensure the maximum von Mises stress in the solid region is less than $\sigma_{y}$ and hence the constraint is defined as

$$
\sigma_{\max }-1 \leq 0 .
$$

In (13), $\zeta$ is a constant used to evaluate the p-norm. As $\zeta \rightarrow \infty, \sigma_{\max }$ tends to the maximum relaxed stress ratio and the function remains unsmoothed. In contrast, when $\zeta \rightarrow 1$ the function is over-smoothed and $\sigma_{\max }$ gives a poor approximate of the maximum stress ratio. Hence, a good choice of $\zeta$ is necessary to create an adequate balance between the two extremes. For our analysis, we use a continuation method on $\zeta$ which is initially equal to 1 and increased over the first 10 iterations to a value of 10 . Continuation on $\zeta$ is commonly adopted for stress constraints in topology optimization [7,28, as it prevents early convergence at a local minima. A final value of 10 was chosen because it had proved to create viable results which is in agreement with similar works 6, 36. A discussion on the choice $\zeta$ and its effect on results is included in section 5.1

For design problems which require stress constraints we introduce regional stress measures. Stress constraints cannot be imposed on each element individually as the number of constraints will become very large, creating a large computational requirement. This issue is solved by imposing a global stress measure using a p-norm function (13). This method reduces computational expenses but provides poor local control over the stress distribution. As a compromise one may subdivide the design domain into $m$ regions and impose a stress constraint on each region. There are multiple examples of regional stress measures in the literature [6, 30, 45, showing that regional stress measures can be used to improve local stress control with a low number of constraints. 
We adopt a method of defining $m$ regions from previous studies by Le et al. [6]. Here, the regions are defined based on the element's stress ratio (13) during the current iteration, $i$, by first sorting element indices in descending order of their corresponding value of $\bar{\sigma}$.

$$
\left\{e_{1}, e_{2}, \ldots, e_{N_{e}}: \bar{\sigma}_{e_{1}}^{i} \geq \bar{\sigma}_{e_{2}}^{i} \geq \ldots \geq \bar{\sigma}_{e_{N_{e}}}^{i}\right\}
$$

Regions are then defined as follows

$$
\Omega_{k}=\left\{e_{k}, e_{m+k}, e_{2 m+k}, \ldots\right\}, k=1,2, \ldots, m .
$$

In this manner, elements in a single region need not be connected to one another, and the elements that are contained within a region, $k$, are subject to change on each iteration. It is worth noting that as the proposed method can allow evolving of the regions the function may become non-differentiable. This would be most likely to occur in early stages of optimization as when the design converges the regions remain predominately unchanged. However, we experienced no adverse effects in using this method.

\subsection{Sensitivity Analysis}

Sensitivity analysis is conducted to determine how each design variable should change between iterations in order to move towards an optimal design. We require sensitivities of the objective function and all the constraint functions. In these design problems there are many more design variables than constraint functions. This makes the adjoint method ideal for calculating the sensitivities. Taking $\mathscr{F}$ to represent our objective/constraint function, we can express $\mathscr{F}$ in Lagrangian form as follows

$$
\Pi=\mathscr{F}+\boldsymbol{\lambda}^{\mathrm{T}} \mathbf{R}=\mathscr{F}+\left[\boldsymbol{\lambda}_{f}^{\mathrm{T}} \boldsymbol{\lambda}_{p}^{\mathrm{T}}\right]\left[\begin{array}{l}
\mathbf{R}_{f} \\
\mathbf{R}_{p}
\end{array}\right]
$$

where $\boldsymbol{\lambda}$ is a Lagrangen multiplier, whose values must be determined. However, note that for any $\boldsymbol{\lambda}, \Pi=\mathscr{F}$ as we know the residual, $\mathbf{R}$, is equal to 0 (refer to $(5)$ ). This equation can also be represented in terms of free and prescribed degrees of freedom (DOF) denoted by the subscripts $f$ and $p$ respectively. We require the sensitivities of the function with respect to each design variable, which is represented in vector form as the derivative of $\Pi$ with respect to $\boldsymbol{x}$. Using the chain rule and simplifying we obtain the expression

$$
\begin{aligned}
\frac{d \Pi}{d \boldsymbol{x}}= & \frac{\partial \mathscr{F}}{\partial \boldsymbol{x}}+\frac{\partial \mathscr{F}}{\partial \mathbf{u}_{f}} \frac{d \mathbf{u}_{f}}{d \boldsymbol{x}} \\
& +\boldsymbol{\lambda}_{f}^{\mathrm{T}}\left[\frac{\partial \mathbf{R}_{f}}{\partial \boldsymbol{x}}+\frac{\partial \mathbf{R}_{f}}{\partial \mathbf{u}_{f}} \frac{d \mathbf{u}_{f}}{d \boldsymbol{x}}\right] \\
& +\boldsymbol{\lambda}_{p}^{\mathrm{T}}\left[\frac{\partial \mathbf{R}_{p}}{\partial \boldsymbol{x}}+\frac{\partial \mathbf{R}_{p}}{\partial \mathbf{u}_{f}} \frac{d \mathbf{u}_{f}}{d \boldsymbol{x}}+\frac{d \mathbf{f}_{p}}{d \boldsymbol{x}}\right] .
\end{aligned}
$$

Together, $\mathbf{u}_{f}$ and $\mathbf{f}_{p}$ are the solutions to the finite element analysis. Note the difference between $\frac{\partial}{\partial x}$ and $\frac{d}{d x}$ operators, which represent explicit and implicit derivatives respectively. Explicit derivatives capture only direct dependence of the function, whereas the implicit derivatives also capture indirect dependence due to the solution of the equilibrium equation (5). We seek a $\boldsymbol{\lambda}$ that causes all implicit terms to vanish.

This paper will detail the derivation of sensitivities for the stress function, derivations of sensitivities for other objective functions used can be found in previous work [1]. Here, our function is (13) where $\mathscr{F}=\sigma_{\text {max }}$. The explicit derivative of $\sigma_{\max }$ is calculated in (19) while the Lagrangian multipliers are calculated in (20), utilizing the derivative of the stress function with respect to the displacement of free DOFs expressed in (21). Note that in (19) and (21) the suffixes a and b represent homogeneous and FGM cases respectively, and $\sigma_{y, E_{e}}$ is the yield stress for an element evaluated using its local Young's modulus, $E_{e}$.

$$
\begin{aligned}
\frac{\partial \sigma_{\max }}{\partial x}= & \left(x^{\eta} \frac{\bar{\sigma}_{V M}}{\sigma_{y}}\right)^{\zeta}\left(\sum_{e=1}^{N_{e}}\left(x_{e} \eta^{\frac{\bar{\sigma}_{V M}}{\sigma_{y}}}\right)^{\zeta}\right)^{\frac{1}{\zeta}-1} \\
& \cdot\left(\frac{\eta}{x}+\frac{1}{\bar{\sigma}_{V M}} \frac{\partial \bar{\sigma}_{V M}}{\partial x}\right)
\end{aligned}
$$




$$
\begin{aligned}
& \frac{\partial \sigma_{\max }}{\partial x}=\left(x_{0} \eta \frac{\bar{\sigma}_{V M}}{\sigma_{y, E_{e}}}\right)^{\zeta}\left(\sum_{e=1}^{N_{e}}\left(x_{0, e} \eta \frac{\bar{\sigma}_{V M}}{\sigma_{y, E_{e}}}\right)^{\zeta}\right)^{\frac{1}{\zeta}-1} \\
& \cdot\left(\frac{\eta}{x_{0}}+\frac{1}{\bar{\sigma}_{V M}} \frac{\partial \bar{\sigma}_{V M}}{\partial E_{e}} \frac{\partial E_{e}}{\partial x_{0}}-\frac{1}{\sigma_{y, E_{e}}} \frac{\partial \sigma_{y, E_{e}}}{\partial E_{e}} \frac{\partial E_{e}}{\partial x_{0}}\right) \\
& \text { for } x \in \boldsymbol{x}_{0} \\
& =\left(x_{0} \eta \frac{\bar{\sigma}_{V M}}{\sigma_{y, E_{e}}}\right)^{\zeta}\left(\sum_{e=1}^{N_{e}}\left(x_{0, e} \eta \frac{\bar{\sigma}_{V M}}{\sigma_{y, E_{e}}}\right)^{\zeta}\right)^{\frac{1}{\zeta}-1} \\
& \cdot\left(\frac{1}{\bar{\sigma}_{V M}} \frac{\partial \bar{\sigma}_{V M}}{\partial E_{e}} \frac{\partial E_{e}}{\partial x_{1}}-\frac{1}{\sigma_{y, E_{e}}} \frac{\partial \sigma_{y, E_{e}}}{\partial E_{e}} \frac{\partial E_{e}}{\partial x_{1}}\right) \\
& \text { for } x \in \boldsymbol{x}_{1} \\
& \lambda_{p}{ }^{\mathrm{T}}=0 \\
& \boldsymbol{\lambda}_{f}^{\mathrm{T}}=-\frac{\partial \sigma_{\max }}{\partial \mathbf{u}_{f}}\left[\frac{\partial \mathbf{R}_{f}}{\partial \mathbf{u}_{f}}\right]^{-1}=-\frac{\partial \sigma_{\max }}{\partial \mathbf{u}_{f}} \mathbf{K}_{f f}^{-1} \\
& \frac{\partial \sigma_{\max }}{\partial \mathbf{u}_{f}}=\frac{1}{\sigma_{y} \zeta}\left(\sum_{e=1}^{N_{e}}\left(x_{e} \eta \frac{\bar{\sigma}_{V M}}{\sigma_{y}}\right)^{\zeta}\right)^{\frac{1}{\zeta}-1} \\
& \cdot\left(\sum_{e=1}^{N_{e}} x_{e}^{\zeta \eta} \bar{\sigma}_{V M}^{\zeta-1} \frac{\partial \bar{\sigma}_{V M}}{\partial \mathbf{u}_{f}}\right) \\
& \frac{\partial \sigma_{\max }}{\partial \mathbf{u}_{f}}=\frac{1}{\sigma_{y, E_{e}} \zeta}\left(\sum_{e=1}^{N_{e}}\left(x_{0, e} \eta \frac{\bar{\sigma}_{V M}}{\sigma_{y, E_{e}}}\right)^{\zeta}\right)^{\frac{1}{\zeta}-1} \\
& \cdot\left(\sum_{e=1}^{N_{e}} x_{0, e}^{\zeta \eta} \bar{\sigma}_{V M}^{\zeta-1} \frac{\partial \bar{\sigma}_{V M}}{\partial \mathbf{u}_{f}}\right)
\end{aligned}
$$

We can now represent 18 as follows

$$
\begin{aligned}
\frac{d \Pi}{d \boldsymbol{x}} & =\frac{\partial \sigma_{\max }}{\partial \boldsymbol{x}}+\boldsymbol{\lambda}_{p}{ }^{\mathrm{T}} \frac{\partial \mathbf{R}_{p}}{\partial \boldsymbol{x}}+\boldsymbol{\lambda}_{f}^{\mathrm{T}} \frac{\partial \mathbf{R}_{f}}{\partial \boldsymbol{x}} \\
& \Longrightarrow \frac{d \Pi}{d x_{e}}=\frac{\partial \sigma_{\max }}{\partial x_{e}}+\boldsymbol{\lambda}_{e}^{\mathrm{T}}\left(\frac{\partial \mathbf{R}}{\partial \boldsymbol{x}}\right)_{e}
\end{aligned}
$$

\section{Results and Discussion}

This section will detail three topology optimization examples - an L-bracket, a gripper, and a force inverter. All homogeneous problems presented in this study are modelled using the highest modulus material where $E_{0}=200$ GPa and $\sigma_{y}=50 \mathrm{MPa}$. Optimization problems are solved using the method of moving asymptotes (MMA) [11]. 


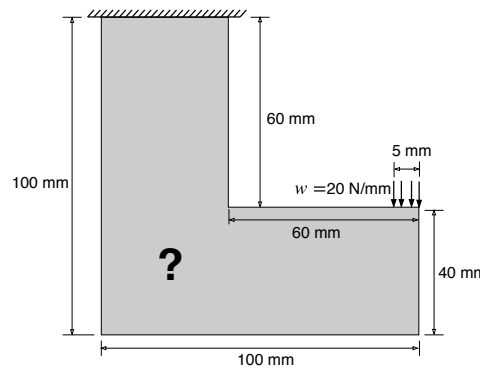

(a)

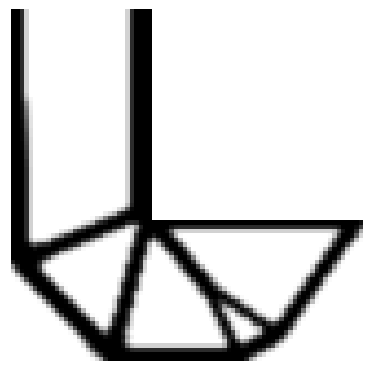

(b)

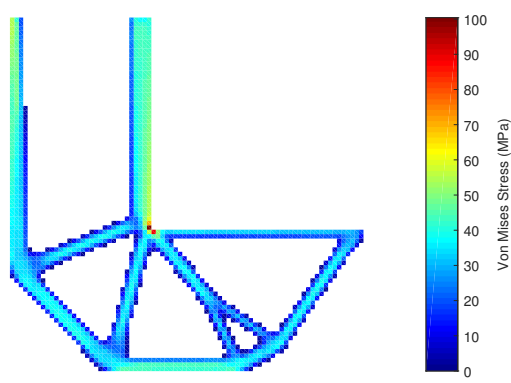

(c)

Fig. 2: (a) L-Bracket design domain, (b) topology solution for compliance minimization problem, and (c) average von Mises stress distribution for compliance minimization problem.

\subsection{L-Bracket}

The first topology optimization problem we will solve is the L-bracket. The L-bracket problem has become the benchmark problem for stress-based topology optimization [4, 5, 38, 31, 29,6, 37]. The design domain is shown in Figure 2a. For comparison purposes we will first solve this as a compliance minimization problem - the most common formulation in topology optimization. The compliance minimization problem is defined as:

$$
\begin{array}{ll}
\min _{x} & : C(\boldsymbol{x})=\mathbf{u}^{T} \mathbf{K u} \\
\text { subject to } & : \int_{\Omega} \frac{M(\boldsymbol{x})}{M_{0}} d \Omega-\bar{M} \leq 0 \\
& : \quad \mathbf{0}<\boldsymbol{x}_{\text {min }} \leq \boldsymbol{x} \leq \mathbf{1}
\end{array}
$$

where $\mathbf{u}$ and $\mathbf{K}$ are the global displacement vector and stiffness matrix respectively; $\int_{\Omega} \frac{M}{M_{0}} d \Omega$ is the resource fraction which is constrained by the maximum value $\bar{M}$. The meaning of the resource fraction will be explained in more detail later, but for now we can treat it as a volume fraction where $M$ is the local contribution to the total volume of the design and $M_{0}$ is the volume of the design domain.

The result of the compliance minimization problem is shown in Figure 2 with a $20 \mathrm{~N} / \mathrm{mm}$ distributed load, the resource fraction constrained at 0.3 , and a filter radius of $1.5 \mathrm{~mm}$. The optimized structure is pictured in Figure $2 \mathrm{~b}$ contains a right angle at the corner of the domain which produces a stress concentration as can be seen in the stress distribution pictured in Figure 2c. The maximum stress in this design was $100.4 \mathrm{MPa}$, roughly double the material's yield strength. The converged structure produced a compliance, $C^{*}$, of 11.39 , this value will help formulate the stress minimization problem.

Our stress minimization problem is defined in (24) where the suffixes (a) and (b) correspond to homogeneous and FGM models respectively.

$$
\begin{gathered}
\min _{x} \quad: \quad \sigma_{\max }(\boldsymbol{x})=\left[\sum_{e=1}^{N_{e}}\left(x_{e} \eta \frac{\bar{\sigma}_{V M}}{\sigma_{y}}\right)^{\zeta}\right]^{\frac{1}{\zeta}} \\
=\left[\sum_{e=1}^{N_{e}}\left(x_{0, e} \eta \frac{\bar{\sigma}_{V M}}{\sigma_{y, E_{e}}}\right)^{\zeta}\right]^{\frac{1}{\zeta}} \\
\text { subject to } \quad: \quad \int_{\Omega} \frac{M(\boldsymbol{x})}{M_{0}} d \Omega-\bar{M} \leq 0 \\
\quad: \quad C-\bar{C} \leq 0 \\
: \quad \mathbf{0}<\boldsymbol{x}_{\min } \leq \boldsymbol{x} \leq \mathbf{1} \\
\\
\quad \mathbf{0}<\boldsymbol{x}_{\min } \leq \boldsymbol{x}_{0} \leq \mathbf{1}, \mathbf{0} \leq \boldsymbol{x}_{1} \leq \mathbf{1}
\end{gathered}
$$




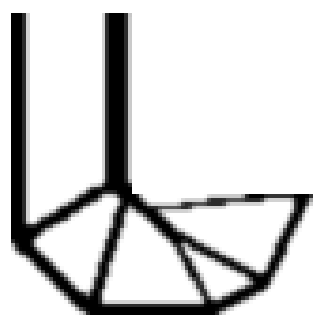

(a)

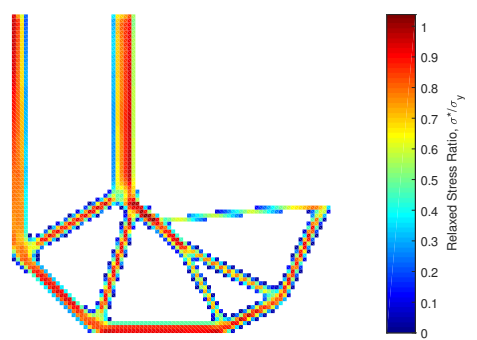

(b)

Fig. 3: Homogeneous L-Bracket stress minimization results: (a) topology solution, and (b) ratio of relaxed stress to yield stress where the maximum relaxed stress is $51.9 \mathrm{MPa}$.

For the stress minimization problem the compliance of the structure, $C$, is constrained by a maximum value of $\bar{C}$. As stress relaxation methods are used we must ensure there is some stiffness in the structure to prevent the optimizer forcing all elements to void in an effort to reduce the stress function. We also want to ensure that the design will perform (with respect to stiffness) to a satisfactory standard, and so the value of the maximum compliance is constrained to $120 \%$ of converged solution to the compliance minimization problem, $C^{*}$.

The resource constraint is treated as a cost function which can be defined in two ways for the FGM designs a volume constraint or a Young's modulus constraint. For the volume constraint $M(x)$ is the local volume of the structure which is a function of the $x_{0}$ variables only, and $M_{0}$ is the volume of the design domain. The assumption with the modulus constraint is that stiffer materials come at a higher cost either due to increased mass or higher procurement cost. Here $M(\boldsymbol{x})$ is the local modulus which is a function of the $x_{0}$ and $x_{1}$ variables, and $M_{0}$ is the sum of local moduli for the entire domain if each element were to have a maximum stiffness, $E_{u}$. For continuity, it is important to note that volume and modulus constraints are equal for homogeneous designs, thus creating a fair basis for comparison. The volume and modulus fractions are calculated using $(25)$ and $(26)$ respectively. Note that (25) and (26) assume a uniform mesh where all elements have an equal volume, as is the case in each example presented.

$$
\begin{gathered}
\int_{\Omega} \frac{M(\boldsymbol{x})}{M_{0}} d \Omega=\frac{1}{N_{e}} \sum_{e=1}^{N_{e}} x_{0, e} \\
\int_{\Omega} \frac{M(\boldsymbol{x})}{M_{0}} d \Omega=\frac{1}{N_{e} E_{u}} \sum_{e=1}^{N_{e}} E_{e}\left(x_{0, e}, x_{1, e}\right)
\end{gathered}
$$

Stress minimization results for the homogeneous L-bracket problem are presented in Figure 3. We can see that the right angle present in the compliance minimization problem has been avoided and instead the topology includes a curved member which eliminates the stress concentration from the design (as seen in Figure 3b). This finding was expected and is in keeping with those presented the literature, thus validating our model. The change in topology of the structure reduces the maximum stress to $51.9 \mathrm{MPa}$. In order to compare stress distributions in the homogeneous design to FGM designs we present distributions of von Mises stress and the relaxed stress ratio with the same contour limits. Our FGM L-bracket problem was solved using a volume constraint where topologies and stress distributions are presented in Figure 4.

FGM designs have a similar topology to the homogeneous compliance minimization problem which ensures the compliance constraint is satisfied, however, more compliant material has been distributed throughout the structure to ensure stress constraints are satisfied. The differences in the $\sigma_{y}-E_{e}$ relationships also affect the Young's modulus distribution. Relationship A (RA), has a near-linear $\sigma_{y}-E_{e}$ interpolation which means more compliant materials are needed to reduce to stresses (refer to Figure 4a). This is because compliant materials are generally able to experience larger strains before reaching before yielding. RA designs have concentrated stiffer material in the two vertical trusses extending from the fixed edge of the design domain, and has distributed lower modulus materials in the rest of the structure. Low-range modulus materials are concentrated towards connections between structural members. To reduce the maximum stress the optimizer has used compliant material immediately below the design domain's right-angle (indicated by the arrow in figure 4a). Compared to the homogeneous design, the maximum ratio of von Mises stress to yield stress has been reduced from 1.04 to 0.86 . 


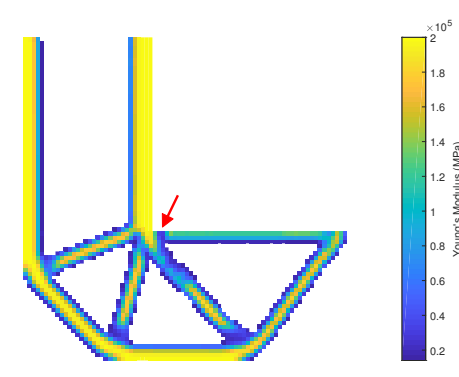

(a) Vol. constrained Rel. A Topology

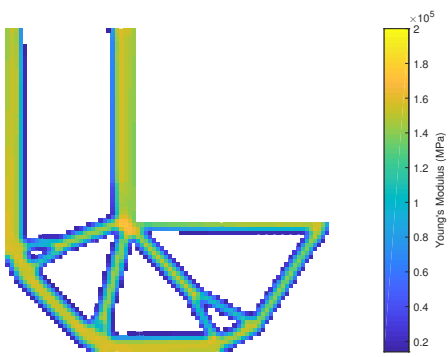

(d) Vol. constrained Rel. B Topology

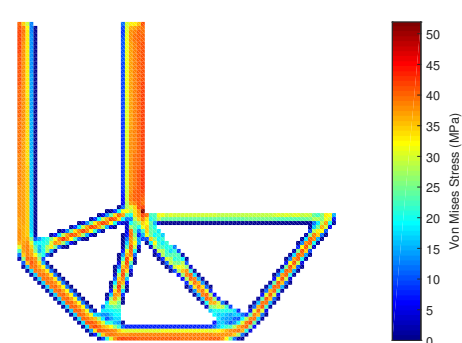

(b) Vol. constrained Rel. A $\bar{\sigma}_{V M}$

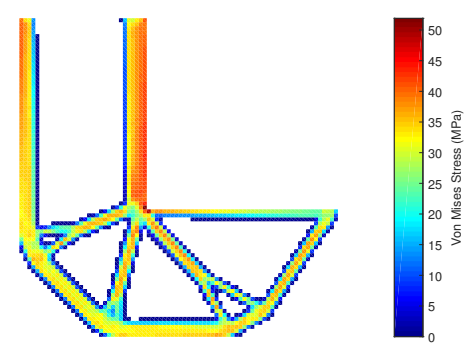

(e) Vol. constrained Rel. B $\bar{\sigma}_{V M}$

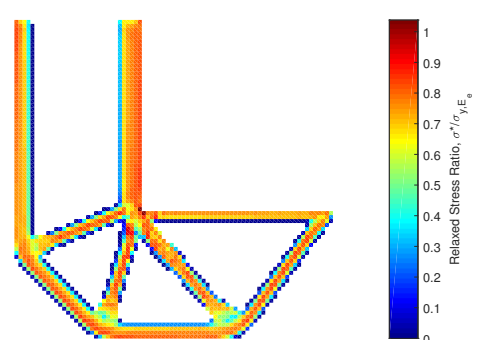

(c) Vol. constrained Rel. A $\bar{\sigma}$

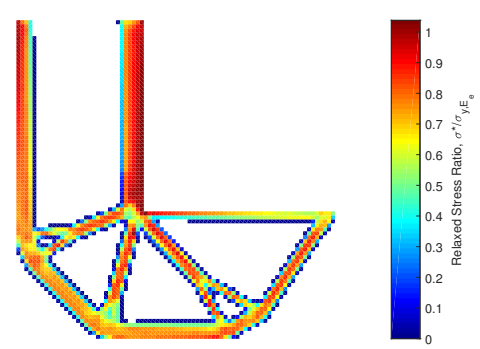

(f) Vol. constrained Rel. B $\bar{\sigma}$

Fig. 4: FGM L-Bracket results. The red arrow in (a) highlights compliant material concentrated at the corner in order to reduce stresses.

Relationship B (RB) is tailored such that midrange modulus materials have high yield strengths and are not susceptible to failure. This gives the optimizer a bias towards mid to high modulus materials. Compared to RA designs, RB designs have opted for more compliant material in structural members but have less variance in Young's modulus throughout the structure. RB designs do not require any low-range modulus materials as stress constraints are easily satisfied. The RB design has greatly improved how the stress is distributed throughout the structure, eliminated stress concentrations, and produced a maximum ratio of relaxed von Mises stress to yield stress of 1.02 .

\subsection{Compliant Mechanisms}

Compliant mechanism designs are produced by maximizing the mechanical advantage. The two design domains studied were: (1) a gripper, and (2) a force inverter. These design domains are shown in Figures 5a and $8 \mathrm{a}$. The optimization problem is defined in (27) where the suffixes (a) and (b) correspond to homogeneous and FGM models respectively. 


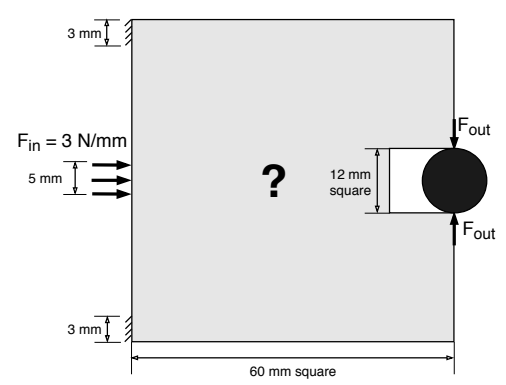

(a)

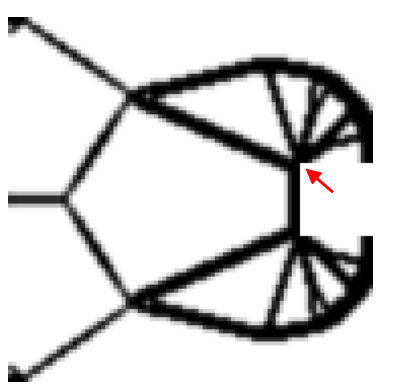

(b)

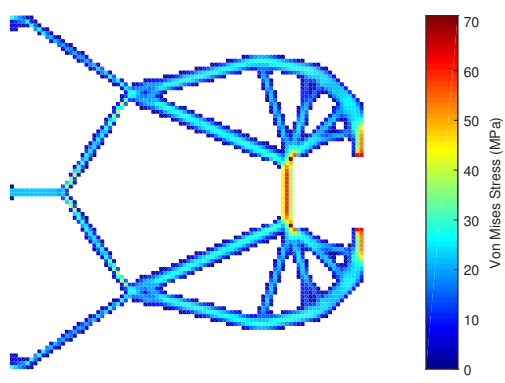

(c)

Fig. 5: (a) Gripper design domain, (b) topology solution with red arrow highlighting a $90^{\circ}$ corner leading to a stress concentration, and (c) average von Mises stress distribution without stress constraints.

$$
\begin{array}{ll}
\min _{x}: \quad f(\boldsymbol{x})=-\frac{F_{\text {out }}}{F_{\text {in }}} \\
\text { subject to }: \quad \int_{\Omega} \frac{M(\boldsymbol{x})}{M_{0}} d \Omega-\bar{M} \leq 0 \\
: \quad C-\bar{C} \leq 0 \\
: \quad\left[\sum_{e \in \Omega_{k}}\left(x_{e}{ }^{\eta} \frac{\bar{\sigma}_{V M}}{\sigma_{y}}\right)^{\zeta}\right]^{\frac{1}{\zeta}} \leq 1, \\
& {\left[\sum_{e \in \Omega_{k}}\left(x_{0, e} \frac{\bar{\sigma}_{V M}}{\sigma_{y, E_{e}}}\right)^{\zeta}\right]^{\frac{1}{\zeta}} \leq 1,2,3, \ldots, m} \\
k=1,2,3, \ldots, m \\
: \quad & \mathbf{0}<\boldsymbol{x}_{\min } \leq \boldsymbol{x} \leq \mathbf{1} \\
& \mathbf{0}<\boldsymbol{x}_{\min } \leq \boldsymbol{x}_{0} \leq \mathbf{1}, \quad \mathbf{0} \leq \boldsymbol{x}_{1} \leq \mathbf{1}
\end{array}
$$

Compliant mechanism designs are solved with both volume and Young's modulus constraints (as defined in (25) and (26) for comparison between the approaches. All compliant mechanisms have a maximum allowable resource fraction, $\bar{M}$, of 0.2 . The second constraint is on the compliance at the input port which ensures a continuous path of material from input to output locations. Without this constraint, the optimizer will push densities to void in an effort to reduce the objective function. The maximum value of compliance, $\bar{C}$, is equal to the compliance of the initial design where all element densities are equal to $\bar{M}$, and $\boldsymbol{x}_{1}=1$ (for the FGM problems). The third constraint is on the max local stresses in $m$ regions as defined in $(16)$, where $m=4$ and $m=5$ for the gripper and inverter designs respectively. Note that different $m$ values were used for the convenience of having the same number of elements in each region - as the two problems had a different number of elements in their finite element mesh, we sought values of $m$ such that $N_{e}$ is divisible by $m$ and $4 \leq m \leq 8$. All designs are subject to an input force of $3 \mathrm{~N} / \mathrm{mm}$.

Results for the homogeneous gripper without and with stress constraints are shown in Figures 5 and 6 respectively. By comparing the gripper results with and without stress constraints we see that stress concentrations at the back of the gripper's jaws have been reduced, note also that this is where the maximum stress occurred in the gripper with no stress constraint. By comparing the topologies in Figures $5 \mathrm{~b}$ and $6 \mathrm{a}$ we see that the optimizer has achieved this reduction in stress by rounding the inner corners of the jaws (as indicated by the arrows). The thickness of the vertical member at the back of the jaws has also been increased and the design contains an extra supporting member extending from the output port. Note that high stresses are still experienced at the output port of the stress-constrained gripper. These stresses are unavoidable for the gripper design problem and are also present in all future results. 


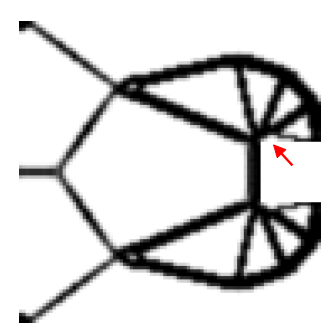

(a)

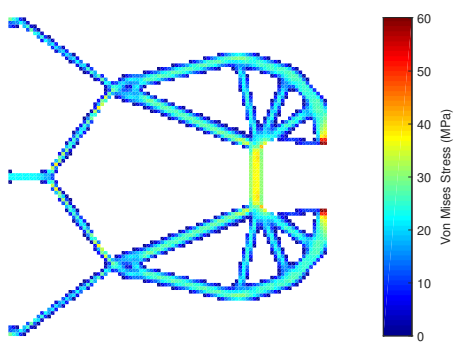

(b)

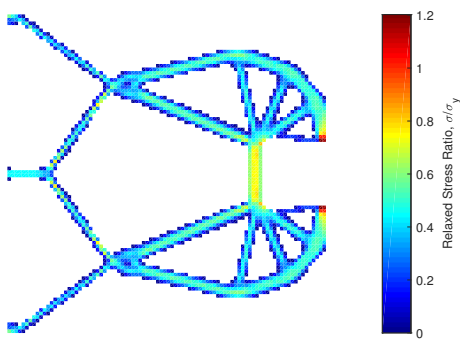

(c)

Fig. 6: Homogeneous stress-constrained gripper results: (a) topology solution with red arrow highlighting the rounded inner edge which avoids stress concentrations, (b) average von Mises stress distribution, and (c) ratio of relaxed stress to yield stress.

FGM gripper designs are shown in Figure 7. There are distinct differences in the topology and Young's modulus distributions between volume- and modulus-constrained designs - as expected, modulus-constrained designs use a larger volume with a wider range of modulus values. This is in keeping with findings in previous work in [1].

The FGM volume-constrained RA gripper (Figures 7a, 7c) has rounded structural members at the top and bottom of the jaws which has eliminated stress concentrations from the design (indicated by the arrow in figure $7 \mathrm{a}$ ). This is a similar result to the homogeneous design in Figure 6a. From Figure $7 \mathrm{c}$ we see that this design has distributed the stresses throughout the structure. The modulus-constrained RA gripper (Figures $7 \mathrm{~d}+7 \mathrm{f}$ ) still contains some stress concentrations but at a lower magnitude than previous designs (and far less than the yield stress). These stress concentrations are all at the compliant joints (and the unpreventable output port) rather than the back of the jaws which was the failure point in the unconstrained gripper design. As stress concentrations are present this also means that stresses are not evenly distributed throughout the structure which is seen in Figure $7 \mathrm{f}$

The FGM volume-constrained RB gripper can withstand higher stresses than its RA counterpart. Similar to the L-bracket results we see that the RA design opts for more of the stiffest material with low modulus materials only concentrated towards the joints. The RB volume-constrained design has concentrated the stiffest material towards the output port and used more mid-range modulus materials throughout the structure. This mechanism employs two vertical structural members behind the jaws in an effort to reduce stress concentrations (as indicated by the arrow in figure $7 \mathrm{~g}$ ). The presence of this additional member reduces the stresses in the primary member including the point of maximum stress (top and bottom of primary structural member). Regions of high stress do not necessarily correspond to high stress-ratio this is evident in comparing Figures $7 \mathrm{~h}$ and $7 \mathrm{i}$ in particularly at joints behind the gripper's jaws.

The material distribution of modulus-constrained designs has few differences between RA and RB, however there is more stiffer material concentrated towards the input and output ports for RB which leads to a higher mechanical advantage. Much of the same conclusions drawn from the RA modulus-constrained gripper can be said for the RB gripper also: low-magnitude stress concentrations are present at compliant joints but not at the back of the jaws; stresses are not distributed throughout the structure; magnitude of stress-ratio is reduced compared to homogeneous design; and mechanical advantage is higher compared to volume constrained designs. A comparison of the mechanical advantage $\left(\frac{F_{\text {out }}}{F_{\text {in }}}\right)$ for all converged designs is shown in Table 1

Table 1: Mechanical advantage of converged gripper designs.

\begin{tabular}{|l||c|}
\hline Design Problem & Mech. Advantage \\
\hline Homogeneous & 4.29 \\
\hline Stress-constrained Homog. & 4.01 \\
\hline FGM RA Volume-constrained & 3.33 \\
\hline FGM RA Modulus-constrained & 3.53 \\
\hline FGM RB Volume-constrained. & 4.08 \\
\hline FGM RB Modulus-constrained & 5.66 \\
\hline
\end{tabular}




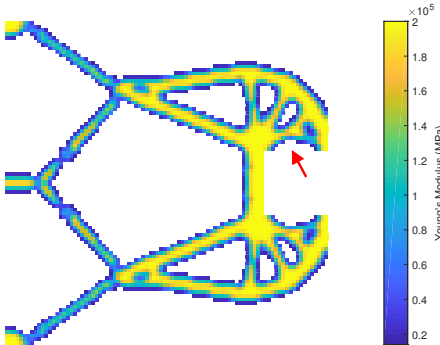

(a) Vol. constrained Rel. A Topology

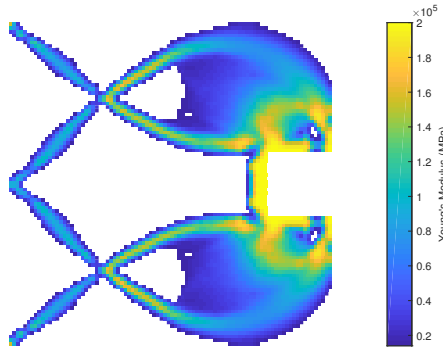

(d) Mod. constrained Rel. A Topology

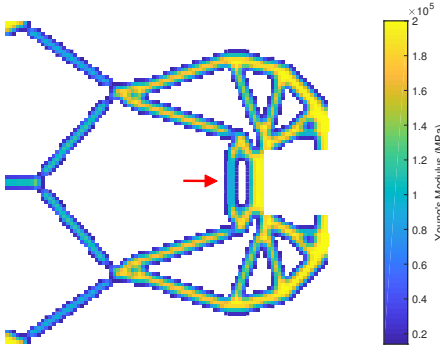

(g) Vol. constrained Rel. B Topology

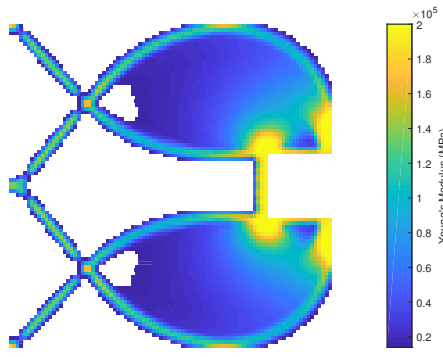

(j) Mod. constrained Rel. B Topology

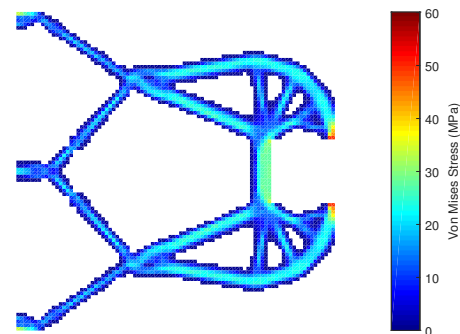

(b) Vol. constrained Rel. A $\bar{\sigma}_{V M}$

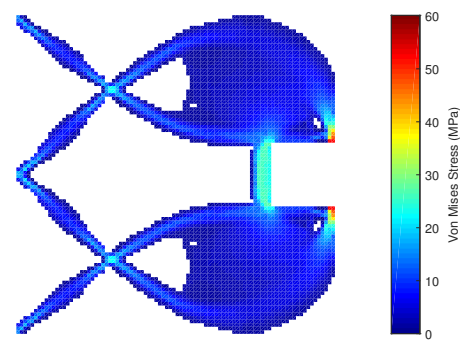

(e) Mod. constrained Rel. A $\bar{\sigma}_{V M}$

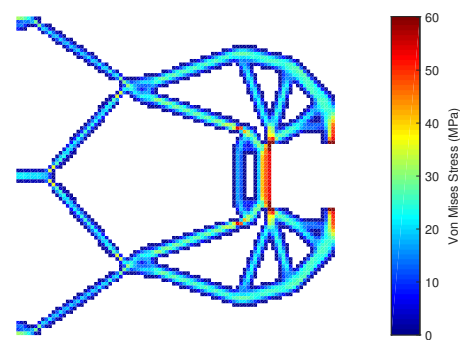

(h) Vol. constrained Rel. B $\bar{\sigma}_{V M}$

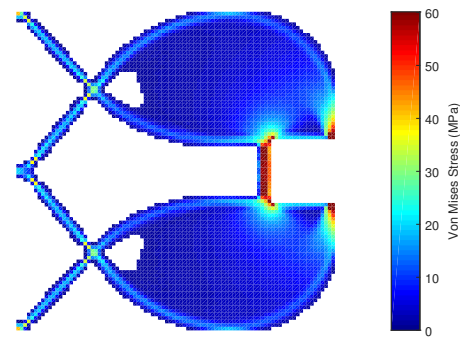

(k) Mod. constrained Rel. B $\bar{\sigma}_{V M}$

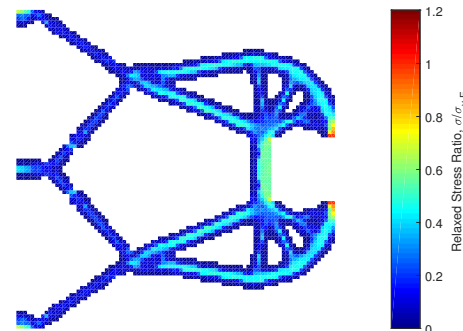

(c) Vol. constrained Rel. A $\bar{\sigma}$

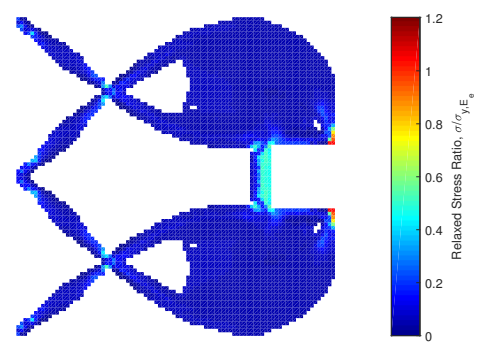

(f) Mod. constrained Rel. A $\bar{\sigma}$

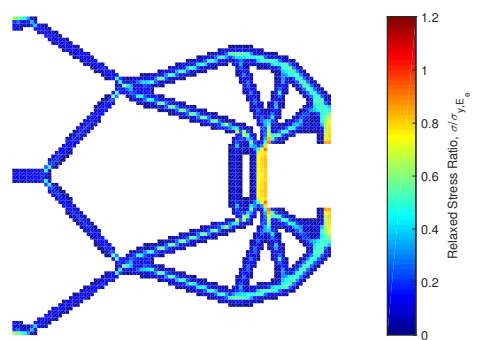

(i) Vol. constrained Rel. B $\bar{\sigma}$

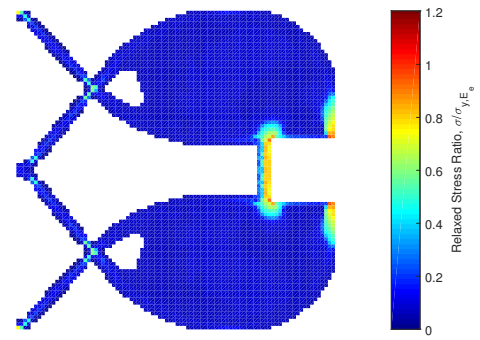

(1) Mod. constrained Rel. B $\bar{\sigma}$

Fig. 7: FGM gripper results. The red arrows in (a) highlight the rounded inner edge and (g) the second vertical member which are used to reduce the stress ratio.

Comparing to the results presented in [1] we see similarities in the topology and material distribution, especially for modulus-constrained designs. However comparing the stress distributions we see that the results of the current study are far more efficient at distributing stresses throughout the structure leading to a reduction in stress concentrations. Thus the proposed method has been able to better distribute stresses in the structure making the design less susceptible to failure.

The optimizers approach to distributing material is distinctly different between RA and RB designs. RA designs have a more even stress distribution, whereas RB designs are able withstand higher stresses so the optimizer is less inclined to distribute stresses throughout the structure. Although some localized stresses are present in the designs, they have been reduced in magnitude or spread over a larger area. It should be also noted that the stress constraint is satisfied for all designs. RB designs have achieved a higher mechanical advantage than RA designs. This is because the $E_{e}-\sigma_{y}$ relationship is tailored such that RB designs are less reliant on more compliant material 


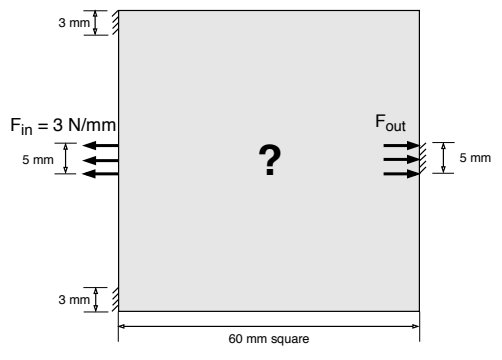

(a)

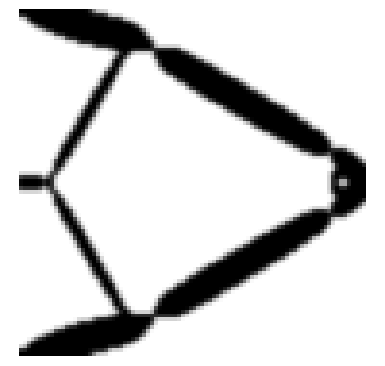

(b)

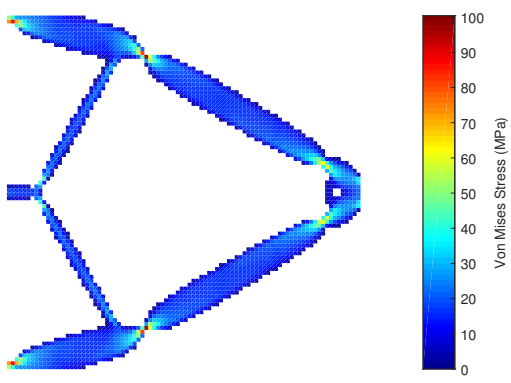

(c)

Fig. 8: (a) Inverter design domain, (b) topology solution problem, and (c) average von Mises stress distribution without stress constraints.

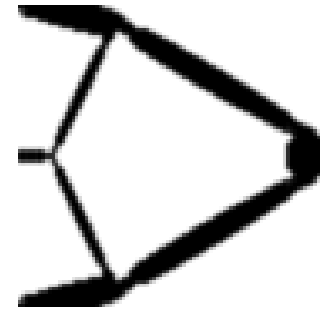

(a)

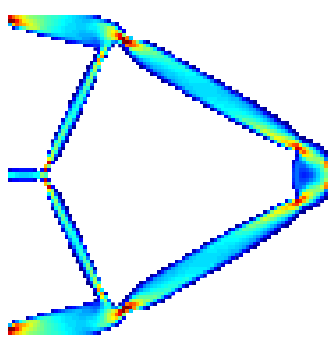

(b)
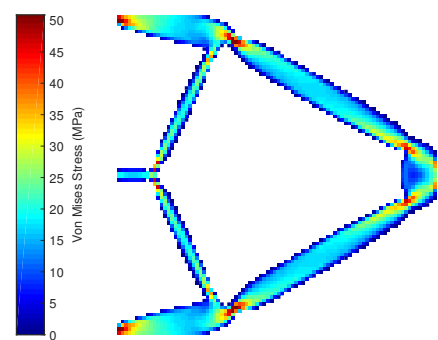

(c)

Fig. 9: Homogeneous stress-constrained inverter results: (a) topology solution, (b) average von Mises stress distribution, and (c) ratio of relaxed stress to yield stress.

to reduce stresses. As stiffer material is used, the design can transmit higher loads and achieve a larger mechanical advantage.

The second compliant mechanism problem studied is the force inverter problem shown in Figure 8a The problem is defined the same as the gripper mechanism in (27). All inverter mechanisms have a resource constraint of 0.2 and the maximum compliance, $\bar{C}$, is equal to the compliance of the initial material distribution where $x_{e}=\bar{M}=0.2$ for all $e$. The results for the homogeneous inverter problem without and with stress constraints are shown in Figures 8 and 9 respectively. The unconstrained results show clear stress concentrations at the joints where there is a maximum stress of $100.1 \mathrm{MPa}$. These compliant joints are very thin and only one element thick at the point of maximum stress. The stress constrained design has much thicker joints which reduces the maximum stress to 50.0 MPa. The stresses have been better distributed throughout the structure, however there are still stress concentrations, albeit spanning a larger area than the unconstrained problem. It should also be noted that all designs experience stress concentrations towards the output port and fixed boundaries. Like the gripper, these stress concentrations are unpreventable and inherent to the inverter problem.

FGM force inverter results are shown in Figure 10. Unlike the gripper problem, here there are very few differences in topology and material distribution between the FGM results. This implies that there is one optimal solution to the inverter problem. The similarities in the inverter designs is also reflected in the value of the objective function where each design produces a similar mechanical advantage as shown in Table 2 Modulus-constrained designs have been able to opt for slightly thicker members which gives a small increase in mechanical advantage. Both volume- and modulus-constrained designs predominately contain the stiffest material, however the presence of more compliant material, particularly close to the joints, has helped to improve stress distributions compared to the homogeneous design. 
Table 2: Mechanical advantage of converged inverter designs.

\begin{tabular}{|l||c|}
\hline Design Problem & Mech. Advantage \\
\hline Homogeneous & 4.49 \\
\hline Stress-constrained Homog. & 4.28 \\
\hline FGM RA Volume-constrained & 4.05 \\
\hline FGM RA Modulus-constrained & 4.32 \\
\hline FGM RB Volume-constrained. & 4.63 \\
\hline FGM RB Modulus-constrained & 4.80 \\
\hline
\end{tabular}

RA designs (Figures $10 a \mid 10 f$ incorporate fewer thin joints compared to RB designs (Figures $10 g$, $10 l$ ). This allows stress to be more efficiently distributed leading to fewer stress concentrations. However, all designs have satisfied the stress constraint.

We experienced convergence issues when the inverter problem was solved with higher loads because the stress constraint could not be satisfied. This implies that the joints are crucial to the function of the force inverter.

\section{Additional examples}

\subsection{Choice of p-norm parameter}

It is important to understand how the choice of the p-norm parameter, $\zeta$, can affect the converged designs. Up to now, all the results presented used $\zeta=10$ (with continuation). Figure 11 shows results for the gripper problem (defined in 27 ) and figure $5 a$ for homogeneous stress-constrained designs with $\zeta=5$ and $\zeta=15$. We see that with a low value of $\zeta$ the optimizer has not been able to been able to round the inner edges of the grippers jaws and stress concentrations are still present. On the other hand, $\zeta=15$ produces a similar design to when $\zeta=10$. Here the inner jaws are rounded and stress concentrations are removed. It is also worth noting that higher values of $\zeta$ are thought to be more susceptible to local minima. Figure 12 shows the FGM RA gripper designs with $\zeta=5$ and $\zeta=15$. Comparing to the $\zeta=10$ designs in figure 7 one can make the same observations as the homogeneous grippers.

\subsection{Impact of resource constraint}

The resource constraint acts as a constraint on cost. Two resource constraints are presented in this work -cost proportional to volume, and cost proportional to the Young's modulus. As a third option we modify the resource constraint in 27) such that more compliant materials associated with low values of $x_{1}$ come at a greater cost. This is done by adding a third term to the constraint which creates a bias towards high values of $x_{1}$.

$$
\int_{\Omega} \frac{M(\boldsymbol{x})}{M_{0}} d \Omega-\bar{M}+\alpha \sum_{i=1}^{N_{e}} \frac{1-x_{1}}{N_{e}} \leq 0
$$

where $\alpha$ is a weighting-parameter for controlling the weight of the bias towards higher modulus materials. Figure 13 shows results for the gripper problem for a bias modulus constraint with $\alpha=0.02, \bar{M}=0.2$, and using relationship A. Comparing to results in $7 d$ we see the large regions of compliant material that is present in the unbiased design have been replaced with a stiffer truss-like structures. Stress concentrations are also eliminated from the design and the mechanism produced a mechanical advantage of 3.34, which is slightly less than the 3.53 achieved with the original cost constraint. This is expected as there is now more restriction on the design space.

\section{Conclusion}

In this work we have introduced a novel method for topology optimization of functionally graded structures with stress considerations. Adjoint sensitivities are derived in detail for both homogeneous and FGM p-norm stress functions. Three design problems are studied, the first being the L-bracket problem which is a benchmark problem for stress-based design. Homogeneous results for the L-bracket match the results present in the literature which helps to validate our model. Expanding to FGM designs, the results have shown us that this method behaves as expected with the maximum relaxed stress-ratio in the structure being reduced. Despite this, some areas of high local stress are still present in converged designs. This is especially prominent in the inverter problems, 


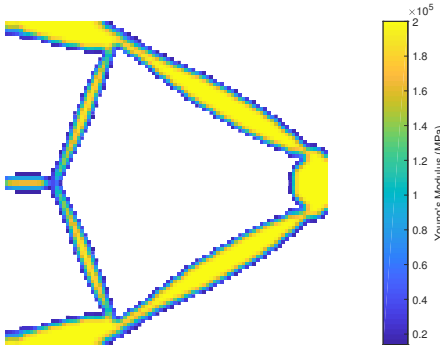

(a) Vol. constrained Rel. A Topology

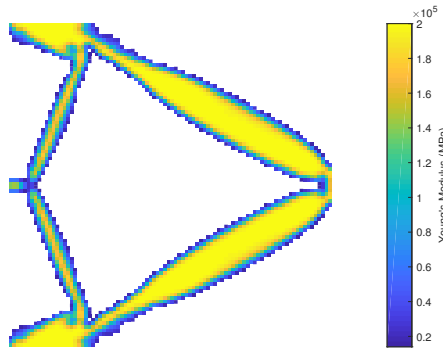

(d) Mod. constrained Rel. A Topology

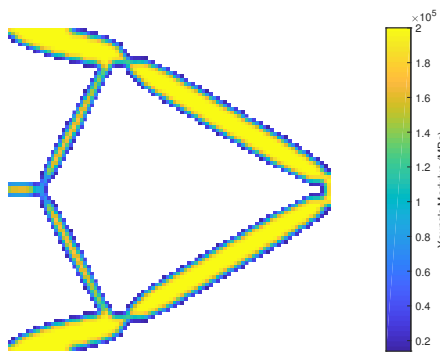

(g) Vol. constrained Rel. B Topology

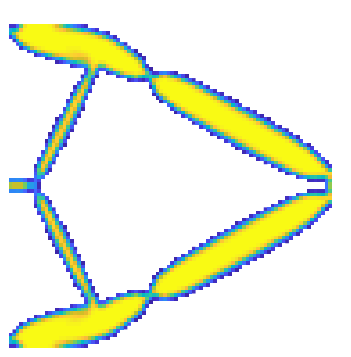

(j) Mod. constrained Rel. B Topology

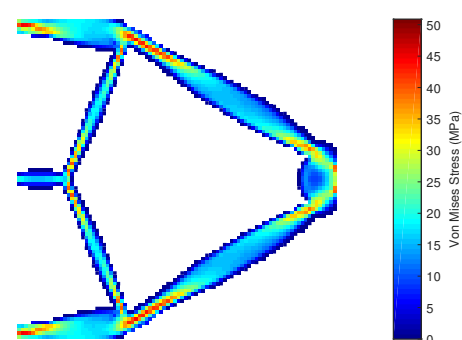

(b) Vol. constrained Rel. A $\bar{\sigma}_{V M}$

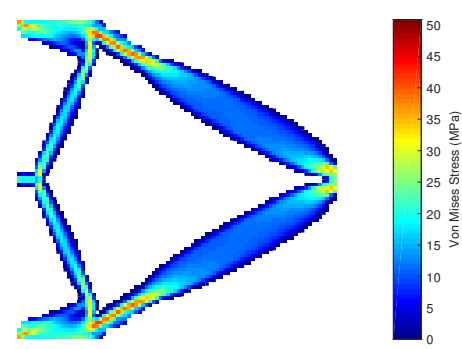

(e) Mod. constrained Rel. A $\bar{\sigma}_{V M}$

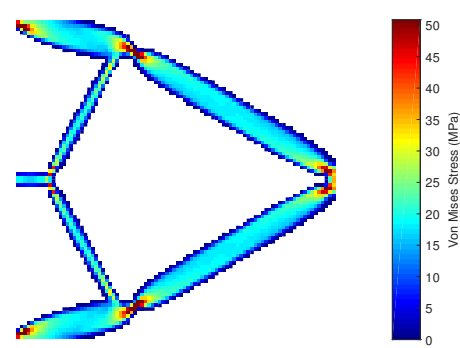

(h) Vol. constrained Rel. B $\bar{\sigma}_{V M}$

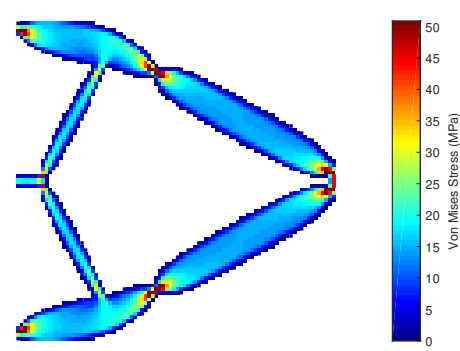

(k) Mod. constrained Rel. B $\bar{\sigma}_{V M}$

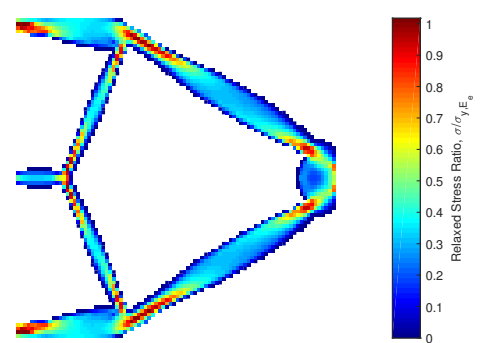

(c) Vol. constrained Rel. A $\bar{\sigma}$

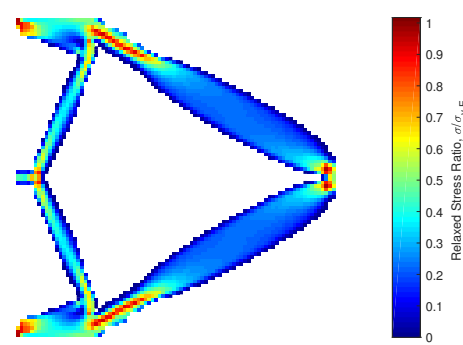

(f) Mod. constrained Rel. A $\bar{\sigma}$

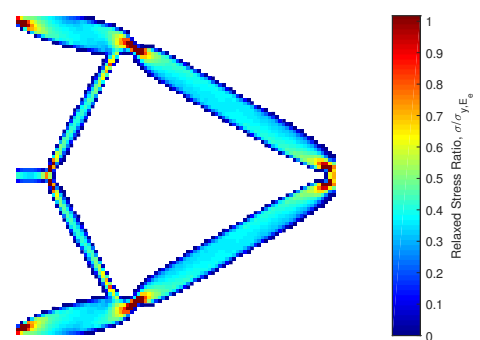

(i) Vol. constrained Rel. B $\bar{\sigma}$

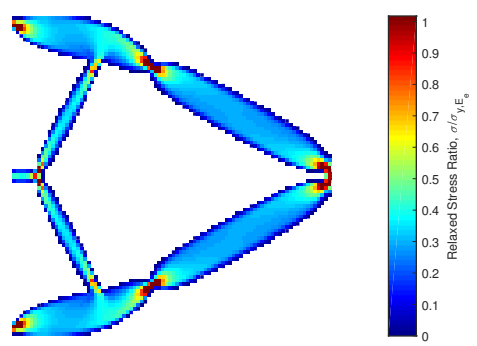

(1) Mod. constrained Rel. B $\bar{\sigma}$

Fig. 10: FGM inverter results.

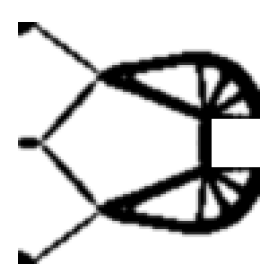

(a) Topology when $\zeta=5$

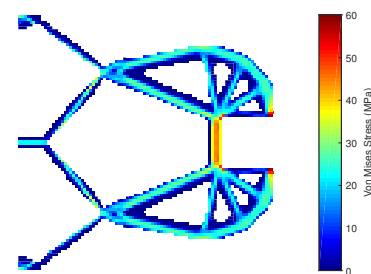

(b) $\bar{\sigma}_{V M}$ when $\zeta=5$

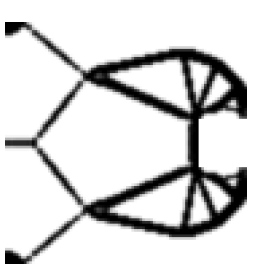

(c) Topology when $\zeta=15$

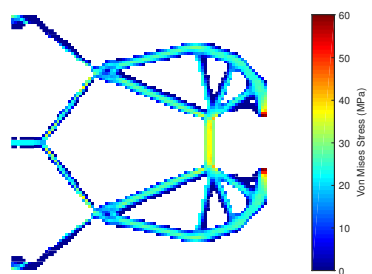

(d) $\bar{\sigma}_{V M}$ when $\zeta=15$

Fig. 11: Homogeneous stress-constrained gripper results with $\zeta=5$ and $\zeta=15$. 


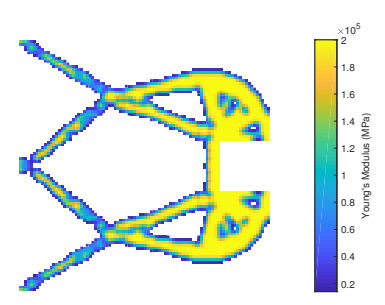

(a) Topology when $\zeta=5$

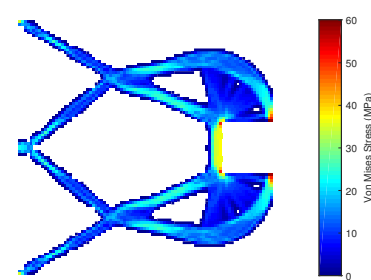

(b) $\bar{\sigma}_{V M}$ when $\zeta=5$

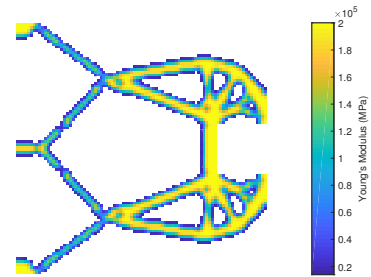

(c) Topology when $\zeta=15$

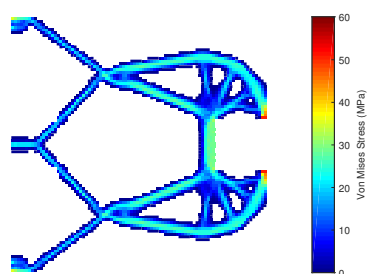

(d) $\bar{\sigma}_{V M}$ when $\zeta=15$

Fig. 12: FGM RA stress and volume-constrained gripper results with $\zeta=5$ and $\zeta=15$.

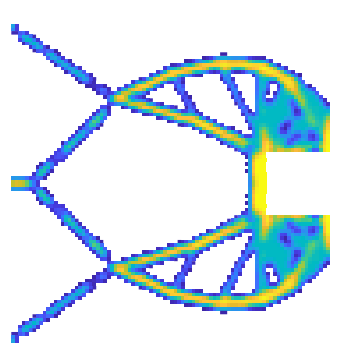

(a)
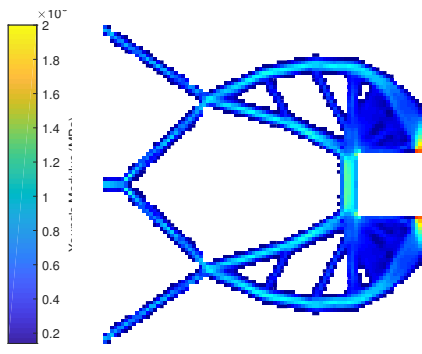

(b)
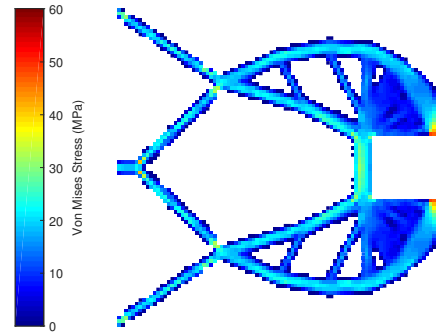

(c)

Fig. 13: Results for a modified cost constraint where

and for higher loads, the inverter problems experienced convergence issues as the designs were unable to satisfy the stress constraint. Comparing volume- and modulus-constrained problems we have observed that stresses are generally lower in modulus-constrained designs but stresses are more evenly distributed in volume-constrained designs. Modulus-constrained compliant mechanism designs achieve the highest mechanical advantage which is in keeping with previous results. RB designs outperform RA designs in each case which shows that for equal resources the design engineer can select a range of materials with favourable material properties to achieve more efficient designs which are not susceptible to failure.

\section{References}

1. Conlan-Smith C, Bhattacharyya A, James KA (2018) Optimal design of compliant mechanisms using functionally graded materials. Struct Multidiscip Optim 57(1):197-212

2. Bruns TE, Totorelli DA (2001) Topology optimization of non-linear structures and compliant mechanisms. Comput Methods Appl Mech Eng 190(26-27):3443-3459

3. Sigmund O (1997) On the design of compliant mechanisms using topology optimization. Mech Struct Mach Int J 25(4):493-542

4. Duysinx P, Bendsøe MP (1998) Topology optimization of continuum structures with local stress constraints. Int J Numer Meth Eng 43(8):1453-1478

5. Duysinx P, Sigmund O (1998) New Developments in Handling Stress Constraints in Optimal Material Distribution. In proceedings of the $7^{\text {th }}$ AIAA/USAF/NASA/ISSMO symposium on multidisciplinary analysis and optimization

6. Le C, Norato J, Bruns T, Ha C, Tortorelli D (2010) Stress-based topology optimization for continua. Struct Multidiscip Optim 41:605-620

7. Lee E, James KA, Martins JRAA (2012) Stress-Constrained Topology Optimization with Design Dependent Loading. Struct Multidiscip Optim 46(5):647-661

8. von Mises R (1913) Mechanik der Festen Körper im Plastisch Deformablen Zustand. Nachrichten von der Gesellschaft der Wissenschaften zu Göttingen 582-592

9. Taylor GI, Quinney H (1931) The Plastic Distortion of Metals. Philosophical Transactions of the Royal Society 230:323-362

10. Tresca H (1864) Sur l'Ecoulement des Corps Solides Soumis a de Fortes Pressions. Comptes Rendus de l'Acadèmie des Sciences vol. $59: 754$

11. Svanberg K (1987) The method of moving asymptotes - a new method for structural optimization. Int J Numer Meth Eng 24(2):359-373

12. Alonso C, Querin OM, Ansola R (2013) A Sequential Element Rejection and Admission (SERA) method for compliant mechanisms design. Struct Multidiscip Optim 47(6):795-807

13. Allaire G, Jouve F, Toader AM (2004) Structural optimization using sensitivity analysis and a level-set method. J Comp Phys 194(1):363393

14. Luo Z, Tong L, Wang MY, Shengin W (2007) Shape and topology optimization of compliant mechanisms using a parameterization level set method. J Comp Phys 227(1):680-705

15. Luo Z, Tong L (2008) A level set method for shape and topology optimization of large-displacement compliant mechanisms. Int J Numer Meth Eng 76(6):862-892

16. Alonso C, Ansola R, Querin OM (2014) Topology synthesis of multi-material compliant mechanisms with Sequential Element Rejection and Admission. Finite Elem Anal Des 85:11-19 
17. Sigmund O (2001) Design of multiphysics actuators using topology optimization -Part II: Two-material structures. Comput Meth Appl Mech 190(49-50):6605-6627

18. Yin L, Ananthasuresh GK (2003) Topology optimization of compliant mechanisms with multiple materials using a peak function material interpolation scheme. Struct Multidiscip Optim 23(1):49-62

19. Wang MY, hen S, Wang X, Mei Y (2005) Design of Multimaterial Compliant Mechanisms Using Level-Set Methods. J Mech Des 127(5):941-956

20. Gaynor A, Meisel NA, Williams CB, Guest JK (2014) Multiple material topology optimization of compliant mechanisms created via polyjet three-dimensional printing. J Manuf Sci Eng 136(6)

21. Wolf D, Yip S (1993) Material interfaces: atomic-level structure and properties, Springer, Netherlands

22. Sigmund O, Torquato S (1997) Design of materials with extreme thermal expansion using a three-phase topology optimization method. J Mech Phys Solids 45(6):1037-1067

23. Stegmann J, Lund E (2005) Discrete material optimization of general shell structures. Int J Numer Methods Eng 62(14):2009-2027

24. Miyamoto Y, Kaysser WA, Rabin BH, Kawasaki A, Ford RG (1999) Functionally graded materials: design processing and applications, Springer

25. Carbonari RC, Silva ECN, Paulino GH (2009) Multi-actuated functionally graded piezoelectric micro-tools design: A multiphysics topology optimization approach. Int J Numer Meth Eng, 77(3):301-336

26. Carbonari RC, Paulino GH, Silva ECN (2010) Integral Piezoactuator with Optimum Placement of Functionally Graded Material - A Topology Optimization Paradigm. J Intel. Material Syst Struct 21(16):1653-1668

27. Matsui K, Terada K (2004) Continuous approximation of material distribution for topology optimization. Int J Numer Meth Eng 59(14):1925-1944

28. Stump FV, Silva ECN, Paulino GH (2007) Optimization of material distribution in functionally graded structures with stress constraints. Commun Numer Methods Eng 23(6):535551

29. Bruggi M, Venini P (2008) A mixed FEM approach to stress constrained topology optimization. Int J Numer Methods Eng 73(11):1693-1714

30. Parìs J, Navarrina F, Colominas I, Casteleiro M (2007) Block aggregation of stress constraints in topology optimization of structures. In: Hernndez S, Brebbia CA (eds) Computer aided optimum design of structures X. Myrtle Beach (SC), USA

31. Parìs J, Navarrina F, Colominas I, Casteleiro M (2009) Topology optimization of continuum structures with local and global stress constraints. Struct Multidisc Optim 39(4):419-437

32. Howell LL (2001) Compliant mechanisms. Wiley.

33. MatWeb Material Property Data, [Online]. Available: https://www.matweb.com/index.aspx.

34. Miyamoto Y, Kaysser WA, Rabin BH, Kawasaki A, Ford RG, Functionally Graded Materials: Design, Processing and Applications, Springer, 1999.

35. Chu S, Gao L, Xiao M, Luo Z, Li H (2017) Stressbased multimaterial topology optimization of compliant mechanisms. Int J Numer Meth Eng 113(7):1021-1044

36. de Leon DM, Alexandersen J, Jun JS, Sigmund O (2015) Stress-constrained topology optimization for compliant mechanism design. Struct Multidisc Optim 52(5):929-943

37. Svanberg K, Werme M (2007) Sequential integer programming methods for stress constrained topology optimization. Struct Multidisc Optim 34(4):277-299

38. Pereira JT, Fancello EA, Barcellos CS (2004) Topology optimization of continuum structures with material failure constraints. Struct Multidisc Optim 26(1-2):50-66

39. Bendsøe MP, Sigmund O (2004) Topology optimization: theory, methods, and applications, Springer

40. Bendsøe MP, Sigmund O (1999) Material interpolation schemes in topology optimization. Arch Appl Math 69:635-654

41. Kirsch U (1990) On singular topologies in optimum structural design. Struct Multidisc Optim 2(3):133-142

42. Rozvany GIN, Sobieszczanski-Sobieski J (1992) New optimality criteria methods: forcing uniqueness of the adjoint strains by cornerrounding at constraint intersections. Struct Multidisc Optim 4(3-4):244-246

43. Rozvany GIN (2001) On design-dependent constraints and singular topologies. Struct Multidisc Optim 21(2):164-172

44. Cheng GD, Guo X (1997) Epsilon-relaxed approach in structural topology optimization. Struct Multidisc Optim 13(4):258-266

45. Jeong SH, Park SH, Choi DH, Yoon GH (2012) Topology optimization considering static failure theories for ductile and brittle materials. Comput Struct 111:116-132

46. Lipton R (2002) Design of functionally graded structures in the presence of stress constraints. Int J Solids Struct 39(9):2575-2586

47. Lipton R, Michael Stuebner (2006) Optimization of composite structures subject to local stress constraints. Comput Methods Appl Mech Eng 196(1-3):66-75 